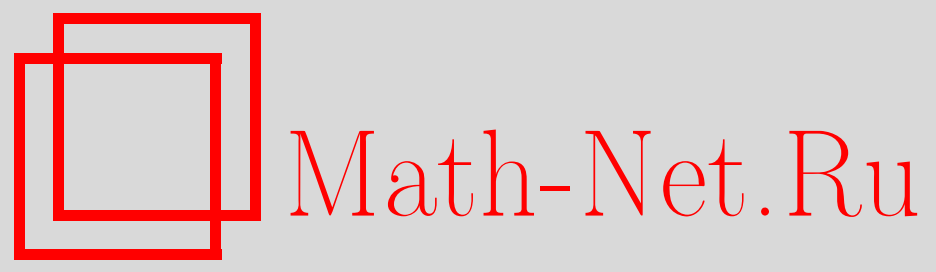

Ю. А. Еловикова, Свойства решетки всех кратно $\Omega$ канонических формаций, Дискрет. матем., 2006, том 18, выпуск 2, 146-158

DOI: https://doi.org/10.4213/dm54

Использование Общероссийского математического портала Math-Net.Ru подразумевает, что вы прочитали и согласны с пользовательским соглашением http: //www . mathnet.ru/rus/agreement

Параметры загрузки:

IP : 52.23 .180 .231

26 апреля 2023 г., 15:01:33

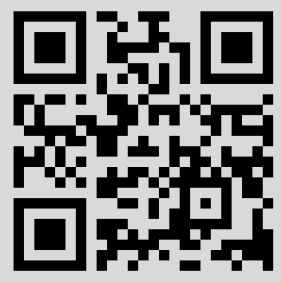




\title{
Свойства решетки всех кратно $\Omega$-канонических формаций
}

\author{
() 2006 г. Ю. А. Еловикова
}

\begin{abstract}
Исследуются решетки $\Omega K_{n}$ и $K_{n}$ соответственно всех $n$-кратно $\Omega$-канонических и $n$-кратно канонических формаций. Основные результаты работы - доказательства ङ-отделимости решетки $\Omega K_{n}$ и совпадения систем тождеств решеток $K_{n}$ и $K_{m}$ при различных целых неотрицательных $n$ и $m$.
\end{abstract}

Исследование $\Omega$-расслоенных формаций с различными направлениями началось после опубликования в 1999 году первых работ В. А. Ведерникова и М. М. Сорокиной, посвященных этой теме. Понятие $\Omega$-расслоенной формации позволило ввести в рассмотрение бесконечное множество новых типов формаций, каждый из которых характеризуется определенным направлением $\varphi$. Следуя [1], через $\Omega$ обозначаем непустой подкласс класса $\mathfrak{I}$ всех конечных простых групп, а направление формации определяется как отображение $\varphi$ класса $\mathfrak{I}$ в множество всех непустых формаций Фиттинга. В частности, в $[1,2]$ были определены канонические ( $K$-формации) и $\Omega$-канонические ( $\Omega K$-формации), имеющие направление $\varphi(A)=\mathscr{G}_{A^{\prime}} \Im_{A}$ при всех $A \in \mathfrak{I}$.

В монографии [3] методы общей теории решеток [4] успешно применяются при исследовании $n$-кратно локальных $\tau$-замкнутых формаций, образующих полную решетку $l_{n}^{\tau}$. Исследуя свойства этой решетки, А. Н. Скиба в [3] доказал совпадение систем тождеств у решеток $l_{n}^{\tau}$ и $l_{m}^{\tau}$ при различных $n$ и $m$. При этом существенно использовалось свойство (s-отделимости решетки $l_{n}^{\tau}$ для любого целого неотрицательного $n$.

Множества $\Omega K_{n}$ и $K_{n}$, соответственно, всех $n$-кратно $\Omega$-канонических и $n$-кратно канонических формаций также образуют полные решетки формаций [5]. Работы автора [5-7] посвящены разработке специального аппарата для применения методов общей теории решеток при изучении $n$-кратно $\Omega$-расслоенных и, в частности, $\Omega K_{n}$-формаций и $K_{n}$-формаций. Основные результаты данной работы - доказательства $\&$-отделимости решетки $\Omega K_{n}$ и совпадения систем тождеств решеток $K_{n}$ и $K_{m}$ при различных целых неотрицательных $n$ и $m$.

Все группы предполагаются конечными. Через \& обозначаем класс всех конечных групп. Необходимые определения и обозначения можно найти в [1-3]. Следуя [1], формацию $\mathfrak{F}$ называем $\Omega$-расслоенной с направлением $\varphi$, если

$$
\mathfrak{F}=\Omega F(f, \varphi)=\left\{G \in G \mid G / O_{\Omega}(G) \in f\left(\Omega^{\prime}\right)\right.
$$

и $G / G_{\varphi(A)} \in f(A)$ для всех $\left.A \in \Omega \cap K(G)\right)$, где $f$ и $\varphi$ - некоторые $\Omega F$-функция и $F R$-функция соответственно. Функцию $f$ называют $\Omega$-спутником формации $\mathfrak{F}$. В част- 
ности, если $\varphi$ - направление $\Omega$-канонической формации, получим $\Omega$-каноническую формацию вида

$$
\mathfrak{F}=\Omega K F(f)=\left(G \in G \mid G / O_{\Omega}(G) \in f\left(\Omega^{\prime}\right)\right.
$$

и $G / O_{A^{\prime}, A}(G) \in f(A)$ для всех $\left.A \in \Omega \cap K(G)\right)$.

Пусть $\theta$ - полная решетка формаций. Будем называть $\Omega$-формационную функщию $\theta$-значной или, коротко, $\Omega \theta$-функцией, если все ее значения принадлежат $\theta$. Через $\Omega F^{\varphi} \theta$ обозначим множество всех $\Omega$-расслоенных формаций с направлением $\varphi$, обладающих хотя бы одним $\Omega \theta$-спутником (в этом случае для $\Omega$-канонических формаций используем обозначение $\Omega K \theta)$. Положим также $\Omega F \theta(\mathfrak{X}, \varphi)=\Omega F^{\varphi} \theta$ form $(\mathfrak{X})$ и, в сокращенном виде, $\Omega K \theta F(\mathfrak{X})$, если $\varphi-$ направление $\Omega$-канонической формации.

Множество всех $\Omega$-расслоенных формаций с направлением $\varphi$ обозначим через $\Omega F^{\varphi}$ ( $\Omega K$ - для множества всех $\Omega$-канонических формаций). Как показано в [5], $\Omega F^{\varphi}$ образует полную решетку формаций. Всякую формацию считают 0 -кратно $\Omega F^{\varphi}$-формацией. Формацию $\mathfrak{F}$ называют $n$-кратно $\Omega F^{\varphi}$-формацией для некоторого натурального $n$, если она обладает $\Omega$-спутником, все непустые значения которого являются $(n-1)$-кратно $\Omega F^{\varphi}$ формациями. Обозначим $\Omega F_{n}^{\varphi}$ множество всех $n$-кратно $\Omega$-расслоенных формаций с направлением $\varphi$. Если $\mathfrak{X}$ - некоторый непустой класс групп, то $\Omega F_{n}(\mathfrak{X}, \varphi)=\Omega F_{n}^{\varphi}$ form $(\mathfrak{X})$ - пересечение всех формаций из $\Omega F_{n}^{\varphi}$, содержащих $\mathfrak{X}$. Аналогично для $\Omega$-канонических формаций используются обозначения $\Omega K_{n}, \Omega K_{n} F(\mathfrak{X})$.

Перейдем к изложению полученных результатов.

Пусть $\mathfrak{X}$ - некоторый непустой класс групп. Полную решетку формаций $\theta$ называют $\mathfrak{X}$-отделимой [3], если для любого терма $\omega\left(x_{1}, x_{2}, \ldots, x_{m}\right)$ сигнатуры $\left\{\cap, \vee_{\theta}\right\}$, любых $\theta$-формаций $\mathfrak{F}_{1}, \mathfrak{F}_{2}, \ldots, \mathfrak{F}_{m}$ и любой группы $A \in \mathfrak{X} \cap \omega\left(\mathfrak{F}_{1}, \mathfrak{F}_{2}, \ldots, \mathfrak{F}_{m}\right)$ найдутся такие $\mathfrak{X}$-группы $A_{1}, A_{2}, \ldots, A_{m}$, что $A \in \omega\left(\theta\right.$ form $A_{1}, \theta$ form $A_{2}, \ldots, \theta$ form $\left.A_{m}\right)$.

Лемма 1. Пусть $\mathfrak{F}=\Omega K_{n} F(\mathfrak{X})$, где $\mathfrak{X}-$ непустой класс групп, $n \geqslant 1$. Если $f-$

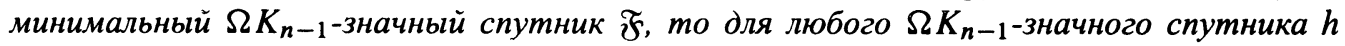
формачии $\mathfrak{F}$

$$
f(A)=\Omega K_{n-1} F\left(B \mid B \in \mathfrak{F} \cap h(A), O_{A}(B)=1\right)
$$

для всех $A \in K(\mathfrak{X}) \cap \Omega$.

Доказательство. Пусть

$$
f_{1}(A)=\Omega K_{n-1} F\left(B \mid B \in \mathfrak{F} \cap h(A), O_{A}(B)=1\right),
$$

где $A \in K(\mathfrak{X}) \cap \Omega$. По следствию 1 из [5]

$$
f(A)=\Omega K_{n-1} F\left(G / O_{A^{\prime}, A}(G) \mid G \in \mathfrak{X}\right)
$$

для всех $A \in \Omega \cap K(\mathfrak{X})$. Поскольку $f(A) \subseteq h(A), f(A) \subseteq \mathfrak{F}$ и $O_{A}\left(G / O_{A^{\prime}, A}(G)\right)=1$ для всех $G \in \mathfrak{X}$, то $\left(G / O_{A^{\prime}, A}(G) \mid G \in \mathfrak{X}\right) \subseteq f_{1}(A)$ и $f(A) \subseteq f_{1}(A)$ для любого $A \in \Omega \cap K(\mathfrak{X})$.

Пусть теперь $A \in \Omega \cap K(\mathfrak{X}), B \in \mathfrak{F} \cap h(A)$ и $O_{A}(B)=1$. Покажем, что $B \in f(A)$. Рассмотрим регулярное сплетение $G=A$ 乙 $B=[K] B, K=A \times \ldots \times A$. По свойствам сплетения из равенства $O_{A^{\prime}}(G) \cap K=1$ следует, что $O_{A^{\prime}}(G)=1$ и $O_{A^{\prime}, A}(G)=O_{A}(G)$. Ясно, что $K \subseteq O_{A}(G)$. Так как $O_{A}(G) \cap B \in G_{A}$, то $O_{A}(G) \cap B \subseteq O_{A}(B)=1$ и $O_{A}(G) \cap B=1$. Следовательно, $O_{A}(G)=O_{A^{\prime}, A}(G)=K$ и $G / O_{A^{\prime}, A}(G) \cong B$.

С другой стороны, $G / O_{A}(G) \in \mathfrak{F}$ и $G / O_{A^{\prime}, A}(G)=G / O_{A}(G) \in h(A)$. Тогда, по лемме 2 из [2], $G \in \Omega K F(h)=\mathfrak{F}$. Отсюда $G / O_{A^{\prime}, A}(G) \in f(A)$, то есть $B \in f(A)$. Следовательно, $\left(B \mid B \in \mathfrak{F} \cap h(A), O_{A}(B)=1\right) \subseteq f(A)$ и $f_{1}(A) \subseteq f(A)$. Таким образом, $f_{1}(A)=f(A)$ для любых $A \in \Omega \cap K(\mathfrak{X})$.

Лемма доказана. 
Лемма 2. Пусть

$$
\mathfrak{F}=\Omega K F(f), \quad G^{\mathfrak{F}} \neq 1, \quad G / O_{\Omega}(G) \in f\left(\Omega^{\prime}\right)
$$

Тогда существует группа $B \in K\left(G^{\mathfrak{F}}\right) \cap \Omega$ такая, что $G / O_{B^{\prime}, B}(G) \notin f(B)$.

Доказательство. Поскольку $G^{\mathfrak{F}} \neq 1$, существует группа $B \in \Omega \cap K(G)$ такая, что $G / O_{B^{\prime}, B}(G) \notin f(B)$. Так как $G / G^{\mathfrak{F}} \in \mathfrak{F}$, учитывая включение $G^{\mathfrak{F}} \in \mathcal{G}_{C^{\prime}}$, получаем, что для всех $C \in\left(K(G) \backslash K\left(G^{\mathfrak{F}}\right)\right) \cap \Omega$

$$
\left(G / G^{\mathfrak{F}}\right) / O_{C^{\prime}, C}\left(G / G^{\mathfrak{F}}\right)=\left(G / G^{\mathfrak{F}}\right) /\left(O_{C^{\prime}, C}(G) / G^{\mathfrak{F}}\right) \cong G / O_{C^{\prime}, C}(G) \in f(C) .
$$

Значит, $B \in K\left(G^{\mathfrak{F}}\right)$. Лемма доказана.

Лемма 3. Если $f$ - внутренний $\Omega$-спутник $\Omega K$-формачии $\mathfrak{F}, G / O_{\Omega}(G) \in f\left(\Omega^{\prime}\right)$ u $G / O_{B}(G) \in f(B)$ для некоторого $B \in \Omega$, то $G \in \mathfrak{F}$.

Доказательство. Так как $G / O_{B}(G) \in f(B) \subseteq \mathfrak{F}$, справедливы соотношения

$$
G^{\mathfrak{F}} \subseteq O_{B}(G), \quad K\left(G^{\mathfrak{F}}\right)=(B) \subseteq \Omega
$$

В силу леммы 2 из соотношения

$$
G / O_{B^{\prime}, B}(G) \cong\left(G / O_{B}(G)\right) /\left(O_{B^{\prime}, B}(G) / O_{B}(G)\right) \in f(B)
$$

следует, что $G^{\mathfrak{F}}=1$ и $G \in \mathfrak{F}$.

Лемма доказана.

Лемма 4. Пусть $G$ - группа с $O_{B}(G)=1$ для некоторой простой группы $B u$

$$
\operatorname{Soc}(G)=N_{1} \times \ldots \times N_{t}, \quad t \geqslant 1 .
$$

Если $M_{i}$ - наибольшая содержашая $N_{1} \times \ldots \times N_{i-1} \times N_{i+1} \times \ldots \times N_{t}$, но не содержашая $N_{i}, i=1, \ldots, t$, нормальная в $G$ подгруппа, то

(1) $G / M_{i}$ монолитична. ее монолит $N_{i} M_{i} / M_{i} G$-изоморфен $N_{i}$ и $O_{B}\left(G / M_{i}\right)=1$,

(2) $M_{1} \cap \ldots \cap M_{t}=1$.

Доказательство. Докажем первое утверждение леммы. Пусть $G / M_{i}$ не монолитична и $T / M_{i} \cdot \triangleleft G / M_{i}, N_{i} M_{i} / M_{i} \cdot \triangleleft G / M_{i}$. Тогда

$$
N_{i} \nsubseteq T, \quad N_{1} \times \ldots \times N_{i-1} \times N_{i+1} \times \ldots \times N_{t} \subseteq M_{i} \subseteq T .
$$

Но в этом случае, по определению $M_{i}, T \subseteq M_{i}$ и $T=M_{i}$. Отсюда следует, что $G / M_{i}$ - монолитическая группа с монолитом $N_{i} M_{i} / M_{i}$. Так как $M_{i} \cap N_{i}=1$, справедливо соотношение

$$
N_{i} M_{i} / M_{i} \cong N_{i} / N_{i} \cap M_{i}=N_{i}
$$

Пусть $L / M_{i}=O_{B}\left(G / M_{i}\right) \neq 1$. Тогда монолит $N_{i} M_{i} / M_{i} \triangleleft L / M_{i}$ и $N_{i} M_{i} / M_{i} \cong N_{i}$ $B$-группа, откуда $N_{i} \subseteq O_{B}(G)=1, N_{i}=1$ и монолит $N_{i} M_{i} / M_{i}=1$, что невозможно. 
Докажем второе утверждение леммы. Предположим, что $M_{1} \cap \ldots \cap M_{t}=K$ и $R$ - минимальная нормальная подгруппа группы $G$ такая, что $R \subseteq K$. Тогда $R \neq N_{i}$, $i=1,2, \ldots, t$. Несложно показать, что

$$
\bigcap_{i=1}^{i} N_{1} \times \ldots \times N_{i-1} \times N_{i+1} \times \ldots \times N_{t}=1 .
$$

Значит, сушествует $s \in\{1,2, \ldots, t\}$ такое, что

$$
R \nsubseteq Q=N_{1} \times \ldots \times N_{s-1} \times N_{s+1} \times \ldots \times N_{t} .
$$

Понятно, что $R Q \subseteq M_{s}$. Покажем, что тогда $N_{s} \subseteq M_{s}$. Поскольку $R Q \subseteq \operatorname{Soc}(G)$, но $R Q \nsubseteq Q$, существует элемент $r=n_{1} \ldots n_{s-1} n_{s} n_{s+1} \ldots n_{t} \in R Q$ такой, что $n_{i} \in N_{i}$, $i=1,2, \ldots, t, n_{s} \neq 1$. Для элемента $q=n_{1}^{-1} \ldots n_{s-1}^{-1} n_{s+1}^{-1} \ldots n_{t}^{-1} \in Q$ справедливо соотношение $1 \neq n_{s}=r q \in R Q \cap N_{s}$. Так что,

$$
R Q \cap N_{s} \neq 1, \quad N_{s} \subseteq R Q \cap N_{s}
$$

Получаем включения

$$
N_{s} \subseteq R Q \subseteq M_{s}
$$

что невозможно по определению $M_{s}$. Полученное противоречие показывает, что $K=1$.

Лемма доказана.

Лемма 5. Пусть $f$-внутренний $\Omega$-спутник $\Omega$-канонической формачии $\mathfrak{F}, R$-монолит группы $A$ и $K(R)=(B) \in \Omega$. Если $A / O_{B^{\prime}, B}(A) \in f(B)$ и $A / O_{\Omega}(A) \in f\left(\Omega^{\prime}\right)$, то $A \in \mathfrak{F}$.

Доказательство. Ясно, что

$$
R \subseteq O_{B^{\prime}, B}(A)=O_{B}(A), \quad A / O_{B}(A) \in f(B) \subseteq \mathfrak{F}
$$

Так как $A / O_{B}(A) \in \mathfrak{F}$ и $C \in K\left(A / O_{B}(A)\right)$, для всех $C \in \Omega \cap K(A), C \not B$,

$$
\begin{aligned}
A / O_{C^{\prime}, C}(A) & \cong\left(A / O_{B}(A)\right) /\left(O_{C^{\prime}, C}(A) / O_{B}(A)\right) \\
& =\left(A / O_{B}(A)\right) / O_{C^{\prime}, C}\left(A / O_{B}(A)\right) \in f(C) .
\end{aligned}
$$

С учетом условия леммы $A / O_{C^{\prime}, C}(A) \in f(C)$ для любых $C \in \Omega \cap K(A)$. Кроме того, вновь по условию леммы $A / O_{\Omega}(A) \in f\left(\Omega^{\prime}\right)$ и по определению $\Omega K$-формации $A \in \mathfrak{F}$.

Лемма доказана.

Лемма 6. Пусть $\mathfrak{M}$ - полуформачия и $A \in \Omega K_{n} F(\mathfrak{M})$. Если $O_{B}(A)=1$ для некоторого $B \in \Omega$, то $A \in \Omega K_{n} F\left(\mathfrak{M}_{1}\right)$, где

$$
\mathfrak{M}_{1}=\left(G / O_{B}(G) \mid G \in \mathfrak{M}\right)
$$

Доказательство. Если $A \in \mathfrak{M}$, то $A \cong A / O_{B}(A) \in \mathfrak{M}_{1}$. Поэтому далее считаем, что $A \notin \mathfrak{M}$.

Пусть $A$ - монолитическая группа с монолитом $R$.

При $n=0$ получаем, что $A \in \Omega K_{0} F(\mathfrak{M})=$ form $M$. По следствию 1.2 .26 из [3] в form $\mathfrak{M}$ существует группа $H \mathrm{c} 2 t+2, t \geqslant 2$, нормальными подгруппами $N, M, N_{1}, \ldots, N_{t}$, 
$M_{1}, \ldots, M_{t}$ такими, что $H / N \cong A, M / N=\operatorname{Soc}(H / N), N_{1} \cap \ldots \cap N_{t}=1$ и $H / N_{i}$-монолитическая $\mathfrak{M}$-группа с монолитом $M_{i} / N_{i}$, который $H$-изоморфен $M / N, i=1, \ldots, t$.

Из равенства $O_{B}(A)=1$ следует, что $O_{B}\left(H / N_{i}\right)=1$. В противном случае монолит $M_{i} / N_{i}$ группы $H / N_{i}$ является $B$-группой и $M_{i} / N_{i} \subseteq O_{B}\left(H / N_{i}\right)$, откуда

$$
M_{i} / N_{i} \cong M / N=\operatorname{Soc}(H / N) \cong \operatorname{Soc}(A) \in \Im_{B},
$$

что противоречит условию $O_{B}(A)=1$. Значит, из включения $H / N_{i} \in \mathfrak{M}$ следует, что $\left(H / N_{i}\right) / O_{B}\left(H / N_{i}\right) \in \mathbb{M}_{1}$ и $A \in H R_{0}\left\{H / N_{1}, \ldots, H / N_{t}\right\} \subseteq$ form $\mathfrak{M}_{1}$.

Пусть теперь $n>0$ и $K(R)=(C)$. Ясно, что $C \not B$. В случае $C \in \Omega$ для любой групшы $G$ выполняется соотношение

$$
G / O_{C^{\prime}, C}(G) \cong\left(G / O_{B}(G)\right) /\left(O_{C^{\prime}, C}(G) / O_{B}(G)\right)=\left(G / O_{B}(G)\right) / O_{C^{\prime}, C}\left(G / O_{B}(G)\right) .
$$

Если $f$ и $h-$ минимальные $\Omega K_{n-1}$-значные спутники $\mathfrak{F}=\Omega K_{n} F(\mathfrak{M})$ и $\mathfrak{S}=\Omega K_{n}\left(\mathfrak{M}_{1}\right)$ соответственно, то

$$
\begin{aligned}
f(C) & =\Omega K_{n-1} F\left(G / O_{C^{\prime}, C}(G) \mid G \in \mathfrak{M}\right) \\
& =\Omega K_{n-1} F\left(G / O_{C^{\prime}, C}(G) \mid G \in \mathfrak{M}_{1}\right)=h(C) .
\end{aligned}
$$

Также с учетом включения $B \in \Omega$ находим, что

$$
G / O_{\Omega}(G) \cong\left(G / O_{B}(G)\right) /\left(O_{\Omega}(G) / O_{B}(G)\right)=\left(G / O_{B}(G)\right) / O_{\Omega}\left(G / O_{B}(G)\right)
$$

и

$$
\begin{aligned}
f\left(\Omega^{\prime}\right) & =\Omega K_{n-1} F\left(G / O_{\Omega}(G) \mid G \in \mathfrak{M}\right) \\
& =\Omega K_{n-1} F\left(G / O_{\Omega}(G) \mid G \in \mathfrak{M}_{1}\right)=h\left(\Omega^{\prime}\right)
\end{aligned}
$$

Поскольку $A \in \mathfrak{F}$, для $C \in \Omega \cap K(A)$ получаем, что $A / O_{C^{\prime}, C}(A) \in f(C)=h(C)$, $A / O_{\Omega}(A) \in f\left(\Omega^{\prime}\right)=h\left(\Omega^{\prime}\right)$ и по лемме $3 A \in \mathcal{H}$.

В случае $C \notin \Omega$ из $A \in \mathfrak{F}$ следует, что

$$
A \cong A / O_{\Omega}(A) \in f\left(\Omega^{\prime}\right)=h\left(\Omega^{\prime}\right) \subseteq \mathcal{H} .
$$

Рассмотрим случай

$$
\operatorname{Soc}(A)=N_{1} \times \ldots \times N_{t}, \quad t>1 .
$$

Пусть $M_{i}$ - наибольшая в $A$ подгруппа, содержащая $N_{1} \times \ldots \times N_{i-1} \times N_{i+1} \times \ldots \times N_{t}$, но не содержашая $N_{i}, i=1, \ldots, t$. Тогда по лемме $4 A \in R_{0}\left(A / M_{1}, \ldots, A / M_{t}\right)$, где $A / M_{i}$ - монолитическая группа с $O_{B}\left(A / M_{i}\right)=1, i=1, \ldots, t$. Зная также, что $A / M_{i} \in \Omega K_{n} F(\mathbb{M})$, по доказанному выше, можно сделать вывод, что $A / M_{i} \in \Omega K_{n} F\left(\mathfrak{M}_{1}\right), i=1, \ldots, t$. Таким образом, $A \in R_{0}\left(A / M_{1}, \ldots, A / M_{t}\right) \subseteq \Omega K_{n} F\left(\mathfrak{M}_{1}\right)$.

Лемма доказана.

Теорема 1. Для любого челого неотричательного п решетка $\Omega K_{n}$ всех $n$-кратно $\Omega$-канонических формачий $\&$-отделима. 
Доказательство. Пусть $\omega\left(x_{1}, \ldots, x_{m}\right)$ - терм сигнатуры $\left\{\cap, \vee_{\Omega K_{n}}\right\}, \mathfrak{F}_{1}, \ldots, \mathfrak{F}_{m}$ принадлежат $\Omega K_{n}$ и $A \in \omega\left(\mathfrak{F}_{1}, \ldots, \mathfrak{F}_{m}\right)$. Индукцией по числу $r$ вхождений символов из $\left\{\cap, \vee_{\Omega K_{n}}\right\}$ в терм $\omega$ покажем, что существуют такие группы $A_{i} \in \mathfrak{F}_{i}, i=1, \ldots, m$, что $A \in \omega\left(\mathfrak{M}_{1}, \ldots, \mathfrak{M}_{m}\right)$, где $\mathfrak{M}_{i}=\Omega K_{n} F\left(A_{i}\right), i=1, \ldots, m$.

При $r=0$ очевидно, что если $A \in \mathfrak{F}$, то $A \in \Omega K_{n} F(A)$.

Пусть $r=1$, то есть $A \in \mathfrak{F}_{1} \cap \mathfrak{F}_{2}$ или $A \in \mathfrak{F}_{1} \vee_{\Omega K_{n}} \mathfrak{F}_{2}$. В случае $A \in \mathfrak{F}_{1} \cap \mathfrak{F}_{2}$ справедливо включение $A \in \Omega K_{n} F(A) \cap \Omega K_{n} F(A)$. В случае $A \in \mathfrak{F}_{1} \vee_{\Omega K_{n}} \mathfrak{F}_{2}$ для доказательства применим индукцию по $n$.

Предположим, что $n=0$, тогда

$$
A \in \mathfrak{F}_{1} \vee_{\Omega K_{0}} \mathfrak{F}_{2}=\mathfrak{F}_{1} \vee \mathfrak{F}_{2}=\operatorname{form}\left(\mathfrak{F}_{1} \cup \mathfrak{F}_{2}\right)=H R_{0}\left(\mathfrak{F}_{1} \cup \mathfrak{F}_{2}\right)
$$

Значит, $A \cong S / N, S \in R_{0}\left(\mathfrak{F}_{1} \cup \mathfrak{F}_{2}\right)$, откуда заключаем, что $S^{\mathfrak{F}_{1}} \cap S^{\mathfrak{F}_{2}}=1$ и $S \in R_{0}\left(S / S^{\mathfrak{F}_{1}}, S / S^{\mathfrak{F}_{2}}\right)$. Таким образом,

$$
\begin{aligned}
A \in \operatorname{form}\left(S / S^{\mathfrak{F}_{1}}, S / S^{\mathfrak{F}_{2}}\right) & =\operatorname{form}\left(S / S^{\mathfrak{F}_{1}}\right) \vee \text { form }\left(S / S^{\mathfrak{F}_{2}}\right) \\
& =\operatorname{form}\left(S / S^{\mathfrak{F}_{1}}\right) \vee_{\Omega K_{0}} \text { form }\left(S / S^{\mathfrak{F}_{2}}\right) .
\end{aligned}
$$

Пусть $n>0$ и утверждение теоремы выполняется для любых натуральных чисел, меньших $n$ и $A \in \mathfrak{F}_{1} \vee_{\Omega K_{n}} \mathfrak{F}_{2}$. Тогда, поскольку по лемме 12 из [5] решетка $\Omega K_{n}$ является $\Omega K$-индуктивной, $A / O_{B_{i}^{\prime}, B_{i}}(A) \in f_{1}\left(B_{i}\right) \vee_{\Omega K_{n-1}} f_{2}\left(B_{i}\right), i=1, \ldots, t$, для всех $B_{i} \in K(A) \cap \Omega=\left(B_{1}, \ldots, B_{t}\right)$, где $f_{1}$ и $f_{2}$ - внутренние $\Omega$-спутники формаций $\mathfrak{F}_{1}$ и $\mathfrak{F}_{2}$ соответственно, а также $A / O_{\Omega}(A) \in f_{1}\left(\Omega^{\prime}\right) \vee \Omega K_{n-1} f_{2}\left(\Omega^{\prime}\right)$. В силу леммы 4 из [1] без потери общности можем считать, что $f_{i}\left(\Omega^{\prime}\right)=\mathfrak{F}_{i}, i=1,2$. По предположению индукции существуют группы $K_{i} \in f_{1}\left(B_{i}\right)$ и $L_{i} \in f_{2}\left(B_{i}\right)$ такие, что

$$
\begin{aligned}
A / O_{B_{i}^{\prime}, B_{i}}(A) \in\left(\Omega K_{n-1} F\left(K_{i}\right)\right) \vee \Omega K_{n-1}\left(\Omega K_{n-1}\right. & \left.F\left(L_{i}\right)\right) \\
& =\Omega K_{n-1} F\left(K_{i}, L_{i}\right), \quad i=1, \ldots, t,
\end{aligned}
$$

и группы $P \in f_{1}\left(\Omega^{\prime}\right), Q \in f_{2}\left(\Omega^{\prime}\right)$ такие, что $A / O_{\Omega}(A) \in \Omega K_{n-1} F(P, Q)$. Пусть $\Omega_{i}$ и $\mathfrak{R}_{i}$ - полуформации, порожденные группами $K_{i}$ и $L_{i}$ соответственно, $i=1, \ldots, t$, тогда

$$
\begin{aligned}
\mathfrak{\Omega}_{i} & =\left(K_{i}^{(1)}, K_{i}^{(2)}, \ldots K_{i}^{(r)}\right), & \mathfrak{L}_{i} & =\left(L_{i}^{(1)}, L_{i}^{(2)}, \ldots, L_{i}^{(s)}\right), \\
\Omega K_{n-1} F\left(K_{i}\right) & =\Omega K_{n-1} F\left(\mathfrak{\Omega}_{i}\right), & \Omega K_{n-1} F\left(L_{i}\right) & =\Omega K_{n-1} F\left(\mathfrak{L}_{i}\right) .
\end{aligned}
$$

Значит,

$$
A / O_{B_{i}^{\prime}, B_{i}}(A) \in \Omega K_{n-1} F\left(K_{i}, L_{i}\right)=\Omega K_{n-1} F\left(\Re_{i} \cup \mathfrak{R}_{i}\right) .
$$

По лемме 6, так как

$$
O_{B_{i}}\left(A / O_{B_{i}^{\prime}, B_{i}}(A)\right)=1 \text {, }
$$

можем считать, что

$$
O_{B_{i}}\left(K_{i}^{(l)}\right)=O_{B_{i}}\left(L_{i}^{(m)}\right)=1, \quad l=1, \ldots, r, \quad m=1, \ldots, s .
$$

Пусть

$$
\begin{aligned}
D_{i} & =K_{i}^{(1)} \times K_{i}^{(2)} \times \ldots \times K_{i}^{(r)}, \\
R_{i} & =L_{i}^{(1)} \times L_{i}^{(2)} \times \ldots \times L_{i}^{(s)} .
\end{aligned}
$$


Поскольку класс $\mho_{B_{i}}-$ формация Фиттинга, согласно утверждению 1.25 гл. 10 в [8] $\mathscr{S}_{B_{i}}$ есть класс Локетта и

$$
O_{B_{i}}\left(D_{i}\right)=O_{B_{i}}\left(K_{i}^{(1)}\right) \times \ldots \times O_{B_{i}}\left(K_{i}^{(r)}\right)=1
$$

аналогично,

$$
O_{B_{i}}\left(R_{i}\right)=1
$$

Кроме того, ясно, что

$$
A / O_{B_{i}^{\prime}, B_{i}}(A) \in \Omega K_{n-1} F\left(\Omega_{i}, \mathfrak{L}_{i}\right)=\Omega K_{n-1} f\left(D_{i}, R_{i}\right) .
$$

Рассмотрим регулярные сплетения

$$
M_{i}=B_{i} \prec D_{i}, \quad N_{i}=B_{i} \prec R_{i}, \quad i=1, \ldots, t .
$$

Отметим, что

$$
O_{B_{i}}\left(M_{i}\right)=B_{i} \times \ldots \times B_{i}
$$

- база сплетения. В противном случае $1 \neq H=O_{B_{i}}\left(M_{i}\right) \cap D_{i} \triangleleft D_{i}$ и $H-B_{i}$-группа, откуда $1 \neq H \subseteq O_{B_{i}}\left(D_{i}\right)=1$, что невозможно. Таким образом,

$$
M_{i} / O_{B_{i}}\left(M_{i}\right) \cong D_{i} \in R_{0} H\left(K_{i}\right) \subseteq f_{1}\left(B_{i}\right)
$$

и, поскольку $B_{i} \in \Omega$,

$$
M_{i} / O_{\Omega}\left(M_{i}\right) \cong\left(M_{i} / O_{B_{i}}\left(M_{i}\right)\right) /\left(O_{\Omega}\left(M_{i}\right) / O_{B_{i}}\left(M_{i}\right)\right) \in f_{1}\left(B_{i}\right) \subseteq \mathfrak{F}_{1}=f_{1}\left(\Omega^{\prime}\right)
$$

Значит, по лемме $3 M_{i} \in \mathfrak{F}_{1}, i=1, \ldots, t$. Аналогично, $N_{i} \in \mathfrak{F}_{2}, i=1, \ldots, t$. Тогда

$$
\begin{aligned}
& A_{1}=M_{1} \times M_{2} \times \ldots \times M_{t} \times P \in \mathfrak{F}_{1}, \\
& A_{2}=N_{1} \times N_{2} \times \ldots \times N_{t} \times Q \in \mathfrak{F}_{2} .
\end{aligned}
$$

Покажем, что

$$
A \in \mathfrak{F}=\left(\Omega K_{n} F\left(A_{1}\right)\right) \vee_{\Omega K_{n}}\left(\Omega K_{n} F\left(A_{2}\right)\right) .
$$

Ясно, что $M_{i} \in \mathfrak{F}$, откуда $M_{i} / O_{B_{i}^{\prime}, B_{i}}\left(M_{i}\right) \in f\left(B_{i}\right)$, где $f$ - внутренний $\Omega$-спутник $\mathfrak{F}$, для которого, согласно лемме 4 из [1], положим $f\left(\Omega^{\prime}\right)=\mathfrak{F}$. Предположим, что $1 \neq O_{B_{i}^{\prime}}\left(M_{i}\right) \triangleleft M_{i}$, тогда $O_{B_{i}^{\prime}}\left(M_{i}\right)$ имеет нетривиальное пересечение с базой $M_{i}$, которая является $B_{i}$-группой, получаем противоречие. Следовательно, $O_{B_{i}^{\prime}}\left(M_{i}\right)=1$ и $O_{B_{i}^{\prime}, B_{i}}\left(M_{i}\right)=O_{B_{i}}\left(M_{i}\right)$ совпадает с базой сплетения $M_{i}$, откуда $M_{i} / O_{B_{i}^{\prime}, B_{i}}\left(M_{i}\right) \cong D_{i}$, то есть $D_{i} \in f^{\prime}\left(B_{i}\right), i=1, \ldots, t$. Аналогично получаем, что $R_{i} \in f\left(B_{i}\right), i=1, \ldots, t$. Тогда $A / O_{B_{i}^{\prime}, B_{i}}(A) \in \Omega K_{n-1} F\left(D_{i}, R_{i}\right) \subseteq f\left(B_{i}\right)$ для любых $B_{i} \in K(A) \cap \Omega$. Группы $P$ и $Q$, как гомоморфные образы $A_{1}$ и $A_{2}$ соответственно, принадлежат $\mathfrak{F}$. Следовательно,

$$
A / O_{\Omega}(A) \in \Omega K_{n-1} F(P, Q) \subseteq \mathfrak{F}=f\left(\Omega^{\prime}\right)
$$

Из доказанного выше вытекает, что

$$
A \in \mathfrak{F}=\left(\Omega K_{n} F\left(A_{1}\right)\right) \vee_{\Omega K_{n}}\left(\Omega K_{n} F\left(A_{2}\right)\right)
$$


и при $r=1$ утверждение леммы выполняется для натурального числа $n$.

Пусть теперь $r>1$, то есть в терм $\omega$ входят $r>1$ символов из $\left\{\cap, \vee \Omega K_{n}\right\}$ и для термов с меньшим числом вхождений утверждение теоремы выполняется. Пусть $\omega$ имеет вид $\omega_{1}\left(x_{i_{1}}, \ldots, x_{i_{a}}\right) \Delta \omega_{2}\left(x_{j_{1}}, \ldots, x_{j_{b}}\right)$, где $\Delta \in\left\{\cap, \vee_{\Omega K_{n}}\right\},\left\{x_{i_{1}}, \ldots, x_{i_{a}}\right\} \cup\left\{x_{j_{1}}, \ldots, x_{j_{b}}\right\}=$ $\left\{x_{1}, \ldots, x_{m}\right\}$. Обозначим через $\mathfrak{R}_{1}$ и $\mathfrak{R}_{2}$ формации $\omega_{1}\left(\mathfrak{F}_{i_{1}}, \ldots, \mathfrak{F}_{i_{a}}\right)$ и $\omega_{2}\left(\mathfrak{F}_{j_{1}}, \ldots, \mathfrak{F}_{j_{b}}\right)$ соответственно. Тогда, по предположению индукции, существуют такие группы $A_{1} \in \mathfrak{R}_{1}$, $A_{2} \in \Omega_{2}$, что $A \in \Omega K_{n} F\left(A_{1}\right) \triangle \Omega K_{n} F\left(A_{2}\right)$. С другой стороны, согласно индукционному предположению, найдутся группы $B_{1}, \ldots, B_{a}, C_{1}, \ldots, C_{b}$ такие, что $B_{k} \in \mathfrak{F}_{i_{k}}$, $k=1, \ldots, a, C_{s} \in \mathfrak{F}_{j_{s}}, s=1, \ldots, b$, и $A_{1} \in \omega_{1}\left(\Omega K_{n} F\left(B_{1}\right), \ldots, \Omega K_{n} F\left(B_{a}\right)\right)$, $A_{2} \in \omega_{2}\left(\Omega K_{n} F\left(C_{1}\right), \ldots, \Omega K_{n} F\left(C_{b}\right)\right)$.

Пусть из множества $x_{i_{1}}, \ldots, x_{i_{a}}$ переменные $x_{i_{1}}, \ldots, x_{i_{t}}$ не входят в слово $\omega_{2}$, a все переменные $x_{i_{t+1}}, \ldots, x_{i_{a}}$ входят в $\omega_{2}$. Рассмотрим группы $D_{i_{k}}=B_{k}$ при $k \leqslant t$, $D_{i_{k}}=B_{k} \times C_{q}$, где $q$ такое, что $x_{i_{k}}=x_{j_{q}}$, при $k>t$. Положим также $D_{j_{s}}=C_{s}$, если $x_{j s} \notin\left\{x_{i_{t+1}}, \ldots, x_{i_{a}}\right\}$. Введем обозначения

$$
\mathfrak{M}_{p}=\Omega K_{n} F\left(D_{i_{p}}\right), \quad \mathfrak{L}_{c}=\Omega K_{n} F\left(D_{j_{c}}\right), \quad p=1, \ldots, a, \quad c=1, \ldots, b .
$$

Понятно, что

$$
A_{1} \in \omega_{1}\left(\Omega K_{n} F\left(B_{1}\right), \ldots, \Omega K_{n} F\left(B_{a}\right)\right) \subseteq \omega_{1}\left(\Omega K_{n} F\left(D_{i_{1}}\right), \ldots, \Omega K_{n} F\left(D_{i_{a}}\right)\right) .
$$

Последнее включение несложно доказать индукцией по числу $s$ вхождений символов из $\left\{\cap, \vee_{\Omega} K_{n}\right\}$ в терм $\omega_{1}$. Действительно, при $s=1$ справедливо включение

$$
\Omega K_{n} F\left(B_{k}\right) \cap \Omega K_{n} F\left(B_{l}\right) \subseteq \mathfrak{M}_{k} \cap \mathfrak{M}_{l},
$$

или

$$
\Omega K_{n} F\left(B_{k}\right) \vee_{\Omega K_{n}} \Omega K_{n} F\left(B_{l}\right) \subseteq \mathfrak{M}_{k} \vee_{\Omega K_{n}} \mathfrak{M}_{l}
$$

При $s>1$ предположим, что включение выполняется для всех натуральных чисел, меньших $s$. Теперь, если терм $\omega_{1}$ имеет $s$ вхождений символов, он имеет вид $\omega_{11} \Delta \omega_{12}$, $\Delta \in\left\{\cap, \vee_{\Omega} K_{n}\right\}$, причем в термы $\omega_{11}$ и $\omega_{12}$ входит меньше, чем $s$ символов из $\left\{\cap, \vee_{\Omega} K_{n}\right\}$. Применяя предположение индукции, получим, что

$$
\begin{aligned}
\omega_{1}\left(\Omega K_{n} F\left(B_{k_{1}}\right), \ldots, \Omega K_{n} F\left(B_{k_{v}}\right)\right) & =\left(\omega_{11} \Delta \omega_{12}\right)\left(\Omega K_{n} F\left(B_{k_{1}}\right), \ldots, \Omega K_{n} F\left(B_{k_{v}}\right)\right) \\
& \subseteq\left(\omega_{11} \Delta \omega_{12}\right)\left(\mathfrak{M}_{k_{1}}, \ldots, \mathfrak{M}_{k_{v}}\right)=\omega_{1}\left(\mathfrak{M}_{k_{1}}, \ldots, \mathfrak{M}_{k_{v}}\right)
\end{aligned}
$$

Итак, $A_{1} \in \omega_{1}\left(\mathfrak{M}_{1}, \ldots, \mathfrak{M}_{a}\right)$. Аналогично можно показать, что $A_{2} \in \omega_{2}\left(\mathfrak{L}_{1}, \ldots, \mathfrak{L}_{b}\right)$. Отсюда следует, что

$$
A \in \Omega K_{n} F\left(A_{1}\right) \triangle \Omega K_{n} F\left(A_{2}\right) \subseteq \omega_{1}\left(\mathfrak{M}_{1}, \ldots, \mathfrak{M}_{a}\right) \Delta \omega_{2}\left(\mathfrak{L}_{1}, \ldots, \mathfrak{L}_{b}\right) .
$$

Выберем формации $\mathfrak{S}_{1}, \ldots, \mathfrak{H}_{m}$ так, что

$$
\begin{array}{rlrl}
\mathfrak{H}_{i_{p}} & =\mathfrak{M}_{p}=\Omega K_{n} F\left(D_{i_{p}}\right), & p=1, \ldots, a ; \\
\mathfrak{H}_{j_{c}}=\mathfrak{R}_{c}=\Omega K_{n} F\left(D_{j_{c}}\right), & c=1, \ldots, b,
\end{array}
$$

тогда

$$
A \in \omega_{1}\left(\mathfrak{S}_{i_{1}}, \ldots, \mathfrak{H}_{i_{a}}\right) \Delta \omega_{2}\left(\mathfrak{H}_{j_{1}}, \ldots, \mathfrak{S}_{j_{b}}\right)=\omega\left(\mathfrak{S}_{1}, \ldots, \mathfrak{S}_{m}\right),
$$

причем $D_{i_{p}} \in \mathfrak{F}_{i_{p}}, D_{j_{c}} \in \mathfrak{F}_{j_{c}}$, что и завершает доказательство теоремы. 
Далее будет рассматриваться множество всех $n$-кратно канонических формаций, образующее полную решетку $K_{n}$. В случае, когда класс простых групा $\Omega$ в определении $\Omega$-расслоенной формации совпадает с классом $\Im$ всех простых групा, используются следующие определения и обозначения [1]: функщию

$$
f: \mathfrak{I} \rightarrow \text { (формации конечных групп), }
$$

принимающую одинаковые значения на изоморфных группах из $\mathfrak{I}$, называют формационной функщией или, коротко, $F$-функщией; формация

$$
\mathfrak{F}=K F(f)=\left(G \in G \mid G / O_{A^{\prime}, A}(G) \in f(A) \text { для всех } A \in \Omega \cap K(G)\right)
$$

называется канонической формацией ( $K$-формацией), а $f-$ ее спутником. Через $K \theta$ будем обозначать множество всех канонических формаций, обладающих хотя бы одним $\theta$-значным спутником. Для произвольного класса групп $\mathfrak{X}$ через $K \theta F(\mathfrak{X})$ и $K_{n} F(\mathfrak{X})$ обозначим формации $K \theta$ form $(\mathfrak{X})$ и $K_{n}$ form $(\mathfrak{X})$ соответственно.

Если $\Sigma \subseteq \mathfrak{I}$ - произвольная совокупность простых групп, используем в дальнейшем обозначение

$$
\mathfrak{D}_{\Sigma}=\bigoplus_{A \in \Sigma} \mathfrak{G}_{A}
$$

Через $\left.K_{n}\right|_{\text {₹ }}$ обозначим решетку всех $K_{n}$-подформаций $n$-кратно канонической формации $\mathfrak{F}$.

Для терма $\omega$ сигнатуры $\left\{\cap, \vee_{\Omega} F_{\varphi} \theta\right\}$ будем обозначать через $\bar{\omega}$ терм сигнатуры $\left\{\cap, \vee_{\theta}\right\}$, получаемый из $\omega$ заменой каждого вхождения символа $\vee_{\Omega} F_{\varphi} \theta$ на $\vee_{\theta}$. В частности, для терма $\omega$ сигнатуры $\left\{\cap, \vee_{\Omega K_{n}}\right\}$ через $\bar{\omega}$ обозначим терм сигнатуры $\left\{\cap, \vee_{\Omega K_{n-1}}\right\}$, получаемый из $\omega$ заменой каждого вхождения символа $\vee \Omega K_{n}$ на символ $\vee \Omega K_{n-1}$.

Лемма 7. Пусть $\theta-\Omega_{\varphi}$-индуктивная решетка формачий, $\omega\left(x_{1}, \ldots, x_{m}\right)-$ терм сигнатуры $\left\{\cap, \vee_{\Omega F_{\varphi} \theta}\right\}, f_{i}$ - внутренний $\theta$-значный спутник $\Omega$-расслоенной формачии $\mathfrak{F}_{i} c$ направлением $\varphi, i=1, \ldots, m$. Тогда

$$
\omega\left(\mathfrak{F}_{1}, \ldots, \mathfrak{F}_{m}\right)=\Omega F\left(\bar{\omega}\left(f_{1}, \ldots, f_{m}\right), \varphi\right) .
$$

Доказательство. Доказательство проведем индукщией по числу $r$ вхождений в терм $\omega$ символов из $\left\{\cap, \vee_{\Omega} F_{\varphi} \theta\right\}$. При $r=0$ лемма, очевидно, верна. Пусть лемма верна для всех натуральных чисел, меньших $r$, и в терм $\omega$ входят $r$ символов операций.

Рассмотрим

$$
\omega\left(x_{1}, \ldots, x_{m}\right)=\omega_{1}\left(x_{i_{1}}, \ldots, x_{i_{a}}\right) \Delta \omega_{2}\left(x_{j_{1}}, \ldots, x_{j_{b}}\right), \quad \Delta \in\left\{\cap, \vee_{\Omega F_{\varphi} \theta}\right\},
$$

и $\left\{x_{i_{1}}, \ldots, x_{i_{a}}\right\} \cup\left\{x_{j_{1}}, \ldots, x_{j_{b}}\right\}=\left\{x_{1}, \ldots, x_{m}\right\}$. По предположению индукции, для термов $\omega_{1}$ и $\omega_{2}$ лемма верна. Тогда

$$
\begin{aligned}
& \omega_{1}\left(\mathfrak{F}_{i_{1}}, \ldots, \mathfrak{F}_{i_{a}}\right)=\Omega F\left(\bar{\omega}_{1}\left(f_{i_{1}}, \ldots, f_{i_{a}}\right), \varphi\right), \\
& \omega_{2}\left(\mathfrak{F}_{j_{1}}, \ldots, \mathfrak{F}_{j_{b}}\right)=\Omega F\left(\bar{\omega}_{2}\left(f_{j_{1}}, \ldots, f_{j_{b}}\right), \varphi\right) .
\end{aligned}
$$

Ясно, что оба спутника $\bar{\omega}_{1}\left(f_{i_{1}}, \ldots, f_{i_{a}}\right)$ и $\bar{\omega}_{2}\left(f_{j_{1}}, \ldots, f_{j_{b}}\right)$ - внутренние спутники соответствующих формаций, значит, по определению $\Omega_{\varphi}$-индуктивной решетки $\theta$,

$$
\begin{aligned}
\omega\left(\mathfrak{F}_{1}, \ldots, \mathfrak{F}_{m}\right) & =\omega_{1}\left(\mathfrak{F}_{i_{1}}, \ldots \mathfrak{F}_{i_{a}}\right) \Delta \omega_{2}\left(\mathfrak{F}_{j_{1}}, \ldots, \mathfrak{F}_{j_{b}}\right) \\
& =\Omega F\left(\bar{\omega}_{1}\left(f_{i_{1}}, \ldots, f_{i_{a}}\right) \bar{\Delta} \bar{\omega}_{2}\left(f_{j_{1}}, \ldots, f_{j_{b}}\right), \varphi\right) \\
& =\Omega F\left(\bar{\omega}\left(f_{1}, \ldots, f_{m}\right), \varphi\right)
\end{aligned}
$$


где $\bar{\Delta}=\vee_{\theta}$, если $\Delta=\vee_{\Omega F_{\varphi} \theta}$. В случае, когда $\Delta=\cap$, справедливо равенство $\bar{\Delta}=\cap$, и утверждение леммы также очевидно.

Лемма доказана.

Следующая лемма вытекает из леммы 12 в [5] и леммы 7.

Лемма 8. Пусть $\omega\left(x_{1}, \ldots, x_{m}\right)-$ терм сигнатуры $\left\{\cap, \vee_{\Omega K_{n}}\right\}, f_{i}-$ внутренний $\Omega K_{n-1}$ значный спутник формачии $\mathfrak{F}_{i}, i=1, \ldots$, m. Тогда

$$
\omega\left(\mathfrak{F}_{1}, \ldots, \mathfrak{F}_{m}\right)=\Omega K F\left(\bar{\omega}\left(f_{1}, \ldots, f_{m}\right)\right)
$$

Теорема 2. Пусть $\Sigma-$ некоторое бесконечное множество простых групn, $\mathfrak{F} \in K_{n}$.

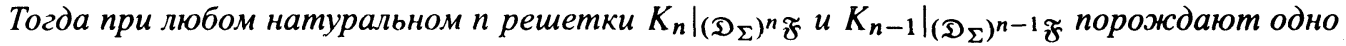
и то же многообразие решеток.

Доказательство. Прежде всего заметим, что для любой простой группы $A \in \mathfrak{I}$ формация $\mathfrak{G}_{A}$ является $n$-кратно канонической формацией со спутником $f$ таким, что $f(A)=\mathfrak{G}_{A}$ и $f(B)=\varnothing$ для всех $A \in \mathfrak{F} \backslash(A)$. Согласно теореме 1 из [6], $\mathfrak{D}_{\Sigma}$ также является $n$-кратно канонической формацией. По следствию 3 из [5], из включений $\mathfrak{F} \in K_{n}$ и $\mathfrak{D}_{\Sigma} \in K_{n}$ для любой простой группы $A \in \Sigma$ следует, что $\left(\mathfrak{D}_{\Sigma}\right)^{n} \mathfrak{F} \in K_{n}$ для любого натурального $n$.

Рассмотрим некоторое тождество

$$
\omega_{1}\left(x_{i_{1}}, \ldots, x_{i_{a}}\right)=\omega_{2}\left(x_{j_{1}}, \ldots, x_{j_{b}}\right)
$$

сигнатуры $\left\{\cap, \vee_{K_{n}}\right\}$. Предположим, что тождество (1) выполняется в решетке $\left.K_{n}\right|_{\left(\mathfrak{D}_{\Sigma}\right)^{n}} \mathfrak{F}$.

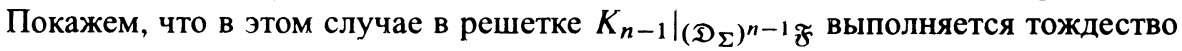

$$
\bar{\omega}_{1}\left(x_{i_{1}}, \ldots, x_{i_{a}}\right)=\bar{\omega}_{2}\left(x_{j_{1}}, \ldots, x_{j_{b}}\right) \text {. }
$$

Ввиду леммы 4.2.4 из [3] для этого достаточно показать, что для произвольных однопорожденных формаций $\mathfrak{F}_{i_{1}}, \ldots \mathfrak{F}_{i_{a}}, \mathfrak{F}_{j_{1}} \ldots, \mathfrak{F}_{j_{h}}$ из $\left.K_{n-1}\right|_{\left(\mathfrak{D}_{\Sigma}\right)^{n-1} \mathfrak{F}}$ справедливо равенство

$$
\bar{\omega}_{1}\left(\mathfrak{F}_{i_{1}}, \ldots, \mathfrak{F}_{i_{a}}\right)=\bar{\omega}_{2}\left(\mathfrak{F}_{j_{1}}, \ldots, \mathfrak{F}_{j_{b}}\right)
$$

Пусть

$$
\mathfrak{F}_{i_{c}}=K_{n-1} F\left(A_{i_{c}}\right) . \quad \mathfrak{F}_{j_{d}}=K_{n-1} F\left(A_{j_{d}}\right), \quad c=1, \ldots, a, \quad d=1, \ldots, b .
$$

Выберем группу $P \in \Sigma$ такую, что $P \notin K\left(A_{i_{1}}, \ldots, A_{i_{a}}, A_{j_{1}}, \ldots, A_{j_{b}}\right)$; пусть $B_{i_{c}}=P \imath A_{i_{c}}$, $B_{j_{d}}=P\left\{A_{j_{d}}\right.$.

Покажем, что формации

$$
\mathfrak{M}_{i_{c}}=K_{n} F\left(B_{i_{c}}\right), \quad \mathfrak{M}_{j_{d}}=K_{n} F\left(B_{j_{d}}\right)
$$

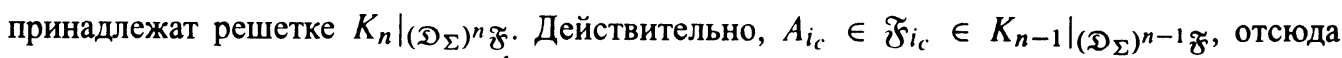
следует, что $A_{i_{c}} \in \mathfrak{F}_{i_{c}} \subseteq\left(\mathfrak{D}_{\Sigma}\right)^{n-1} \mathfrak{F}$. В то же время

$$
B_{i_{c}}=(P \times P \times \ldots \times P) \lambda A_{i_{c}} \in \mathfrak{D}_{\Sigma}\left(\mathfrak{D}_{\Sigma}\right)^{n-1} \mathfrak{F}=\left(\mathfrak{D}_{\Sigma}\right)^{n} \mathfrak{F}
$$

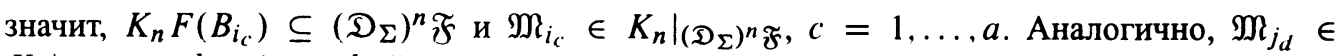

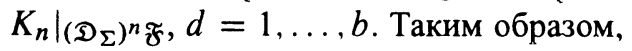

$$
\mathfrak{M}=\omega_{1}\left(\mathfrak{M}_{i_{1}}, \ldots, \mathfrak{M}_{i_{a}}\right)=\omega_{2}\left(\mathfrak{M}_{j_{1}}, \ldots, \mathfrak{M}_{j_{b}}\right)=\mathfrak{H}
$$


Пусть $f_{i_{c}}$ - минимальный $K_{n-1}$-значный спутник $\mathfrak{M}_{i_{c}}, c=1, \ldots, a, f_{j_{d}}$ - минимальный

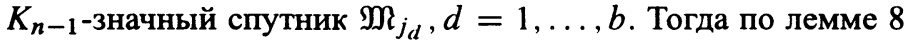

$$
\begin{aligned}
\omega_{1}\left(\mathfrak{M}_{i_{1}}, \ldots, \mathfrak{M}_{i_{a}}\right) & =K F\left(\bar{\omega}_{1}\left(f_{i_{1}}, \ldots, f_{i_{a}}\right)\right), \\
\omega_{2}\left(\mathfrak{M}_{j_{1}}, \ldots, \mathfrak{M}_{j_{a}}\right) & =K F\left(\bar{\omega}_{2}\left(f_{j_{1}}, \ldots, f_{j_{b}}\right)\right) .
\end{aligned}
$$

Рассмотрим минимальные $K_{n-1}$-значные спутники $m$ и $h$ формаций $\mathfrak{M}$ и $\mathfrak{S}$ соответственно. Покажем, что

$$
\bar{\omega}_{1}\left(f_{i_{1}}, \ldots, f_{i_{a}}\right)(P)=m(P) .
$$

Рассмотрим спутник $s=\bar{\omega}_{1}\left(f_{i_{1}}, \ldots, f_{i_{a}}\right)$ и проведем индукцию по числу $r$ вхождений символов из $\left\{\cap, \vee_{K_{n-1}}\right\}$ в терм $\bar{\omega}_{1}$. При $r=1$ в случае $\mathfrak{M}=\mathfrak{M}_{k} \vee_{K_{n}} \mathfrak{M}_{t}$ справедливо соотношение

$$
\begin{aligned}
s(P) & =\left(f_{k} \vee_{K_{n-1}} f_{t}\right)(P) \\
& =\left[K_{n-1} F\left(G / O_{P^{\prime}, P}(G) \mid G \in \mathfrak{M}_{k}\right)\right] \vee_{K_{n-1}}\left[K_{n-1} F\left(G / O_{P^{\prime}, P}(G) \mid G \in \mathfrak{M}_{t}\right)\right] \\
& \subseteq K_{n-1} F\left(G / O_{P^{\prime}, P}(G) \mid G \in \mathfrak{M}_{k} \cup \mathfrak{M}_{t}\right)=m(P)
\end{aligned}
$$

и, в силу минимальности $m$,

$$
s(P)=m(P) \text {. }
$$

В случае $\mathfrak{M}=\mathfrak{M}_{k} \cap \mathfrak{M}_{t}$, поскольку $s=f_{k} \cap f_{t}$ - внутренний спутник $\mathfrak{M}$, по лемме 1

$$
\begin{aligned}
m(P) & =K_{n-1} F\left(H \mid H \in f_{k}(P) \cap f_{t}(P) \cap \mathfrak{M}_{k} \cap \mathfrak{M}_{t}, O_{P}(H)=1\right) \\
& =K_{n-1} F\left(H \mid H \in f_{k}(P) \cap f_{t}(P), O_{P}(H)=1\right) .
\end{aligned}
$$

Так как

$$
H \in f_{k}(P)=K_{n-1} F\left(B_{k} / O_{P^{\prime}, P}\left(B_{k}\right)\right)=K_{n-1} F\left(A_{k}\right) \subseteq \mathcal{G}_{P^{\prime}},
$$

справедливы соотношения

$$
O_{P}(H)=1, \quad m(P)=K_{n-1} F\left(f_{k}(P) \cap f_{t}(P)\right)=f_{k}(P) \cap f_{t}(P)=s(P) .
$$

Пусть утверждение верно для всех натуральных чисел, меньших $r$, и $\bar{\omega}_{1}$ имеет $r$ вхождений символов из $\left\{\cap, \vee_{K_{n-1}}\right\}$. Тогда

$$
\bar{\omega}_{1}\left(f_{i_{1}}, \ldots, f_{i_{a}}\right)=\bar{\omega}_{1}^{\prime}\left(f_{k_{1}}, \ldots, f_{k_{p}}\right) \bar{\triangle} \bar{\omega}_{1}^{\prime \prime}\left(f_{t_{1}}, \ldots, f_{t_{q}}\right)
$$

где $\bar{\Delta} \in\left\{\cap, \vee_{K_{n-1}}\right\}$ и $\left\{f_{k_{1}}, \ldots, f_{k_{p}}\right\} \cup\left\{f_{t_{1}}, \ldots, f_{t_{q}}\right\}=\left\{f_{i_{1}}, \ldots, f_{i_{a}}\right\}$. По предположению

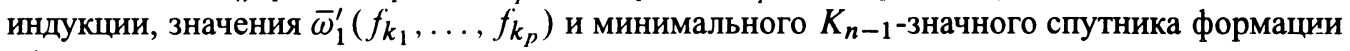
$\omega_{1}^{\prime}\left(\mathfrak{M}_{k_{1}}, \ldots, \mathfrak{M}_{k_{p}}\right)$ на $P$ совпадают. То же можно сказать о значениях на $P$ спутника $\bar{\omega}_{1}^{\prime \prime}\left(f_{t_{1}}, \ldots, f_{t_{q}}\right)$ и минимального $K_{n-1}$-значного спутника формации $\omega_{1}^{\prime \prime}\left(\mathfrak{M}_{t_{1}}, \ldots, \mathfrak{M}_{t_{q}}\right)$. Так как для $r=1$ утверждение справедливо, совпадают и значения на $P$ спутника $\bar{\omega}_{1}^{\prime}\left(f_{k_{1}}, \ldots, f_{k_{p}}\right) \bar{\triangle} \bar{\omega}_{1}^{\prime \prime}\left(f_{t_{1}}, \ldots, f_{t_{q}}\right)=\bar{\omega}_{1}\left(f_{i_{1}} \ldots, f_{i_{a}}\right)$, и минимального $K_{n-1}$-значного спутника $m$ формации

$$
\omega_{1}^{\prime}\left(\mathfrak{M}_{k_{1}}, \ldots, \mathfrak{M}_{k_{p}}\right) \Delta \omega_{1}^{\prime \prime}\left(\mathfrak{M}_{t_{1}}, \ldots, \mathfrak{M}_{t_{q}}\right)=\omega_{1}\left(\mathfrak{M}_{i_{1}}, \ldots, \mathfrak{M} i_{i_{a}}\right)=\mathfrak{M}
$$

Аналогично можно показать, что

$$
\bar{\omega}_{2}\left(f_{j_{1}}, \ldots, f_{j_{b}}\right)(P)=h(P)
$$


Итак, поскольку $\mathfrak{M}=\mathfrak{S}$, справедливо равенство

$$
m(P)=h(P)
$$

и

$$
\bar{\omega}_{1}\left(f_{i_{1}} \ldots, f_{i_{a}}\right)(P)=\bar{\omega}_{2}\left(f_{j_{1}}, \ldots, f_{j_{b}}\right)(P)
$$

В то же время

$$
f_{i_{c}}(P)=K_{n-1} F\left(B_{i_{c}} / O_{P^{\prime}, P}\left(B_{i_{c}}\right)\right)=K_{n-1} F\left(A_{i_{c}}\right)=\mathfrak{F}_{i_{c}}, \quad c=1, \ldots, a,
$$

аналогично,

$$
f_{j_{d}}(P)=\mathfrak{F}_{j_{d}}, \quad d=1, \ldots, b .
$$

Таким образом,

$$
\bar{\omega}_{1}\left(\mathfrak{F}_{i_{1}}, \ldots, \mathfrak{F}_{i_{a}}\right)=\bar{\omega}_{2}\left(\mathfrak{F}_{j_{1}}, \ldots, \mathfrak{F}_{j_{b}}\right),
$$

то есть тождество (2) выполняется для произвольных однопорожденных формаций $\mathfrak{F}_{i_{1}}, \ldots, \mathfrak{F}_{i_{a}}, \mathfrak{F}_{j_{1}}, \ldots, \mathfrak{F}_{j_{b}}$ из решетки $\left.K_{n-1}\right|_{\left(\mathfrak{D}_{\Sigma}\right)^{n-1}}$.

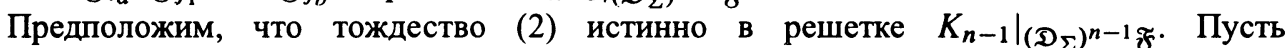
$\mathfrak{F}_{i_{1}}, \ldots, \mathfrak{F}_{i_{a}}, \mathfrak{F}_{j_{1}}, \ldots, \mathfrak{F}_{j_{b}}-$ произвольные формации из $K_{n} \mid\left(\mathfrak{D}_{\Sigma}\right)^{n} \mathfrak{F}, f_{i_{c}}-$ минимальный $K_{n-1}$-значный спутник $\mathfrak{F}_{i_{c}}, c=1, \ldots, a, f_{j_{d}}$ - минимальный $K_{n-1}$-значный спутник $\mathfrak{F}_{j_{d}}, d=1, \ldots, b$. По лемме 8

$$
\begin{aligned}
\omega_{1}\left(\mathfrak{F}_{i_{1}}, \ldots, \mathfrak{F}_{i_{a}}\right) & =K F\left(\bar{\omega}_{1}\left(f_{i_{1}}, \ldots, f_{i_{a}}\right)\right), \\
\omega_{2}\left(\mathfrak{F}_{j_{1}}, \ldots, \mathfrak{F}_{j_{b}}\right) & =K F\left(\bar{\omega}_{2}\left(f_{j_{1}}, \ldots, f_{j_{b}}\right)\right) .
\end{aligned}
$$

Для любой простой группы $A$ значения спутников $f_{i_{c}}(A)$ и $f_{j_{d}}(A)$ принадлежат решетке $\left.K_{n-1}\right|_{\left(\mathfrak{D}_{\Sigma}\right)^{n-1} \mathfrak{F}}, c=1, \ldots, a, d=1, \ldots, b$. Действительно, все непустые значения спутника $f_{i_{c}}$ имеют вид

$$
f_{i_{c}}(A)=K_{n-1} F\left(G / O_{A^{\prime}, A}(G) \mid G \in \mathfrak{F}_{i_{c}}\right) .
$$

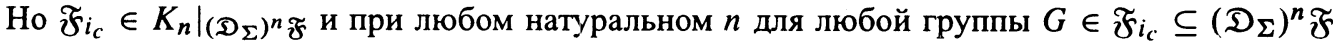
справедливо включение $G^{\left(\mathfrak{D}_{\Sigma}\right)^{n-1} \mathfrak{F}} \in \mathfrak{D}_{\Sigma}$, а поскольку $\mathfrak{D}_{\Sigma} \subseteq \mathfrak{G}_{A^{\prime}} \mathfrak{G}_{A}$ для всех $A \in \mathfrak{I}$, отсюда следует, что $G^{\left(\mathfrak{D}_{\Sigma}\right)^{n-1} \mathfrak{F}} \in \mathfrak{D}_{\Sigma} \subseteq \mathfrak{G}_{A^{\prime}} \mathfrak{G}_{A}$. Следовательно, для произвольной группы $G \in \mathfrak{F}_{i_{c}}$ получаем, что

$$
G / O_{A^{\prime}, A}(G) \cong\left(G / G^{\left(\mathfrak{D}_{\Sigma}\right)^{n-1} \mathfrak{F}}\right) /\left(O_{A^{\prime}, A}(G) / G^{\left(\mathfrak{D}_{\Sigma}\right)^{n-1} \mathfrak{F}}\right) \in\left(\mathfrak{D}_{\Sigma}\right)^{n-1} \mathfrak{F}
$$

значит,

$$
f_{i_{c}}(A) \subseteq\left(G_{\Sigma}\right)^{n-1} \mathfrak{F}
$$

и

$$
\left.f_{i_{c}}(A) \in K_{n-1}\right|_{\left(D_{\Sigma}\right)^{n-1} \mathfrak{F}}, \quad A \in \mathfrak{I}, \quad c=1, \ldots, a .
$$

Аналогично,

$$
\left.f_{j_{d}}(A) \in K_{n-1}\right|_{\left(D_{\Sigma}\right)^{n-1} \mathfrak{F}}, \quad A \in \mathfrak{I}, \quad d=1, \ldots, b .
$$


Таким образом,

$$
\bar{\omega}_{1}\left(f_{i_{1}}, \ldots, f_{i_{a}}\right)(A)=\bar{\omega}_{2}\left(f_{j_{1}}, \ldots, f_{j_{b}}\right)(A)
$$

для любых $A \in \mathfrak{I}$. Наконец, формации, определяемые спутниками $\bar{\omega}_{1}$ и $\bar{\omega}_{2}$, равны:

$$
\omega_{1}\left(\mathfrak{F}_{i_{1}}, \ldots, \mathfrak{F}_{i_{a}}\right)=\omega_{2}\left(\mathfrak{F}_{j_{1}}, \ldots, \mathfrak{F}_{j_{b}}\right)
$$

Справедливость тождества (1) в решетке $\left.K_{n}\right|_{\left(\mathscr{G}_{\Sigma}\right)^{n}}$ ₹ установлена.

Теорема доказана.

Следствие 1. Пусть $\Sigma$ - некоторое бесконечное множество простых групn, $m$ u $n$ челье неотрицательные числа, $m \leqslant n, \mathfrak{S} \in K_{n} u \mathfrak{F}=\mathfrak{G}_{\Sigma} \mathfrak{S}$. Тогда множества тождеств решеток $\left.K_{n}\right|_{\mathfrak{z}}$ и $\left.K_{m}\right|_{\mathfrak{z}}$ совпадают.

Доказательство. Заметим, что

$$
\mathfrak{F}=\mathfrak{G}_{\Sigma} \mathfrak{H}=\left(\mathfrak{D}_{\Sigma}\right)^{n} \mathfrak{F}=\left(\mathfrak{D}_{\Sigma}\right)^{m} \mathfrak{F}
$$

Формация $\mathcal{G}_{\Sigma}$ является $n$-кратно канонической со спутником $f$ таким, что $f(A)=\mathbb{G}_{\Sigma}$ для всех $A \in \Sigma$ и $f(A)=\varnothing$ при $A \notin \Sigma$. Значит, по следствию 3 из [5], $\mathfrak{F} \in K_{n}$. Ввиду теоремы 1 , множества тождеств решеток $\left.K_{n}\right|_{\left(\mathfrak{D}_{\Sigma}\right)^{n} \mathfrak{F}}=\left.K_{n}\right|_{\mathfrak{F}}$ и $\left.K_{m}\right|_{\left(\mathfrak{D}_{\Sigma}\right)^{n} \mathfrak{F}}=\left.K_{m}\right|_{\mathfrak{F}}$ совпадают. Следствие доказано.

Следствие 2. При любых чельх неотричательных $m$ и п множества тождеств решеток $K_{m} u K_{n}$ совпадают.

Доказательство. Можем рассматривать $K_{n}=\left.K_{n}\right|_{G}, K_{m}=\left.K_{m}\right|_{G}$. Выбирая в следствии $1 \mathfrak{H}=\mathscr{G}$ и множество $\Sigma=\mathfrak{I}$ всех конечных простых групп, получаем требуемое утверждение. Следствие доказано.

\section{Список литературы}

1. Ведерников В. А., Сорокина М. М., $\Omega$-расслоенные формации и классы Фиттинга. Дискретная математика 2001 13, №3, 125-144.

2. Ведерников В. А., Максимальные спутники $\Omega$-расслоенных формаций и классов Фиттинга конечных групп. Труды Матем. иль-та им. В. А. Стеклова РАН (2001) 2, 217-233.

3. Скиба А. Н., Алгебра формачий. Беларуская навука, Минск, 1997.

4. Грэтцер Г., Общая теория решеток. Мир, Москва, 1982.

5. Скачкова Ю. А., Решетки $\Omega$-расслоенных формаций. Дискретиая математика (2002) 14, №2, 85-94.

6. Скачкова Ю. А., Булевы решетки кратно $\Omega$-расслоенных формаций. Дискретиая математика (2002) 14, №3, 42-46.

7. Скачкова Ю. А., Решетки $\Omega$-расслоенных формаций. В сб.: Тезисы докл. III Международиой алгебраической конферепции в Украипе. Сумской госпедунив., Сумы, 2001, с. 251.

8. Doerk K., Hawkes T., Finite soluble groups. Gruyter, Berlin, 1992.

Статья поступила 17.05.2004. 